\title{
Nuevas subjetividades sociales e implicancias éticas
}

\author{
Yanina Mariel Ferreyra* \\ Eliana Jaime Bacile**
}

RESUMEN

El presente trabajo analiza la práctica denominada "empresas recuperadas". El mismo toma como caso la "Cooperativa de trabajo de la salud Junín Ltda." de la ciudad de Córdoba. Estudia lo acontecido, los logros, subrayando las relaciones entre la construcción de identidades y de poder. Se plantean consideraciones ético-institucionales y su incidencia en las decisiones de los trabajadores.

Palabras clave: Empresas recuperadas - ética institucional - estrategias de intervención - poder - movimiento social

\section{Novas subjetividades sociais e implicancias éticas}

\begin{abstract}
O presente trabalho analisa a prática denominada "empresas recuperadas". O mesmo toma como caso a "Cooperativa de trabalho da saúde Junín Ltda." da cidade de Córdoba. Estuda o acontecido, os lucros, sublinhando as relações entre a construção de identidades e de poder. Propõem-se considerações ético institucionais e seu incidencia nas decisões dos trabalhadores.
\end{abstract}

Palavras chave: Empresas recuperadas - ético institucionais - estratégias de intervenção - poder- movimento social

\section{New social and ethical implications subjectivities}

\section{ABSTRACT}

This paper analizes the practise named "recovered enterprises". It studies the case of "Cooperativa de Trabajo de la Salud Junín Ltda." from Córdoba city. It focuses on the events, the aims, highlighting the relationships between the construction of identities and of power. Besides, this work raises ethic and institutional issues and its effects on the workers' desitions.

Key Words: Recovered enterprises - institutional ethics - intervention strategies - power - social movement.

* Argentina. Licenciada en Psicología. Facultad de Psicología. Universidad Nacional de Córdoba. Correo electrónico: yani_ferreyra83@hotmail.com

** Argentina. Licenciada en Psicología. Facultad de Psicología. Universidad Nacional de Córdoba. Correo electrónico: eli_bacile@yahoo.com.ar 


\section{Introducción}

En el presente trabajo se intenta transmitir la experiencia realizada en la Cooperativa de Trabajo de la Salud Junín Ltda., guiada por el proyecto-beca "Hacer para transformar. Estrategias de intervención ético-institucional e identidad en la Cooperativa de Trabajo de la Salud Junín Ltda.", presentado ante la Secretaría de Extensión Universitaria de la Universidad Nacional de Córdoba, en el año 2008.

Tal proyecto surge a partir de otro, desarrollado en el Centro de Estudios Avanzados (CEA) de la UNC, durante el 2006-2007, con aval académico de la SECyT-UNC", denominado: “Empresas recuperadas. Procesos identitarios y discursividad en la "Cooperativa de Trabajo de la Salud Junín Ltda." de la Ciudad de Córdoba, Argentina”.

La concreción del proyecto-beca en la Clínica se contextualiza en cierta práctica que, desde hace más de una década, se viene desarrollando en el país, cuyo conjunto se denomina "empresas recuperadas", como parte de lo que se considera "nuevos movimientos sociales".

Entre los años 2001 y 2003, debido a la gran crisis financiera que se desarrolla en el país, muchas empresas son recuperadas por sus trabajadores con el objetivo de defender sus fuentes de trabajo y mantenerlas en funcionamiento frente a la amenaza de quiebre y despidos. En torno a este fenómeno, se abre un conjunto de procesos sociales, dinámicas políticas, estrategias jurídicas y desarrollos económicos que proporcionan a esta problemática una gran complejidad y riqueza. Estas empresas representan uno de los emergentes más originales de la lucha de los trabajadores por conservar sus puestos de trabajo frente a la destrucción del aparato productivo.

Esta recuperación de empresas no ha sido homogénea, por eso da como resultado diferentes formas de puesta en práctica y gestión de las mismas. Sin embargo, estas heterogeneidades no impiden que puedan observarse un conjunto de elementos comunes que las caracterizan como la toma del control de la empresa por parte de los trabajadores, es decir, no hay dueño ni patrón que permita o deje de permitir la participación, sino que la totalidad de los miembros

1 Secretaría de Ciencia y Tecnología de la Universidad Nacional de Córdoba. 
de la empresa asumen su dirección y administración, esto como consecuencia de una serie de episodios que conforman un cuadro de deterioro de la condición salarial, despidos, movilizaciones en torno a la toma de las instalaciones o del aparato productivo, la guardia en carpas frente a la empresa para evitar el retiro de maquinarias o materias primas indispensables para la continuidad de la producción.

Así, a partir de lo investigado durante los años 2006 y 2007, analizando lo acontecido y poniendo el acento en las relaciones entre los aspectos vinculados a la construcción de identidades y las relaciones de poder, categorías que resultaron operativas para el estudio de nuestro objeto, se llevaron a cabo, durante el año 2008, estrategias de intervención dentro de la Clínica que tuvieron como objetivo principal organizar a los trabajadores, a través de la elaboración de un plan de acción, para favorecer el logro del objetivo institucional que se habían propuesto a lo largo de estos cinco años, desde la recuperación de la clínica, el cual plantea: "Retribuir la solidaridad brindada por la sociedad, a través de campañas comunitarias en sectores vulnerables, comedores, merenderos, etcétera"; y ante las diversas dificultades e impedimentos con los que se encontraban para su logro, es que planteamos dicha proyección para permitir a los miembros de la Cooperativa la consecución de diversas campañas comunitarias de manera continua y constante en la ciudad de Córdoba.

De esta manera, la Clínica tuvo la posibilidad de fortalecer lazos sociales y retribuir la solidaridad que recibieron de la sociedad en los momentos de crisis, redimensionando su identidad institucional, favoreciendo el reconocimiento social de una empresa que está al servicio de la salud integral de los sujetos en la ciudad de Córdoba.

\section{La identidad como producto de las relaciones de poder: Implicancias ético- institucionales}

Los acontecimientos de diciembre de 2001, al ritmo de la consigna que "se vayan todos" y los cacerolazos, hechos que pusieron a los ciudadanos de toda Argentina a repensar su posición en la cotidianidad y cuestionaron toda una producción intelectual, que trataba al sujeto argentino, desde la posición que tenía en la década 
del '70, donde el miedo y la parálisis se apoderó de los colectivos; o desde la apatía y el desinterés hacia la participación política y la movilización social que caracterizó a la década del menemismo.

La movilización del 19 y 20 de diciembre de 2001 señala un cambio al respecto. Como señala Lewkowicz, no solo que no había miedo, "debe haber sido el estado de sitio más ridículo de la historia (...) [no sólo se sentía] un tono subjetivo: fiesta, relajación, confianza, alegría" (Lewkowicz 2002:21); sino también la consigna podía ser interpretada con un límite a la clase política, a la "clase de Estado".

En este contexto, los actores sociales debieron responder con nuevas conductas, nuevas formas de resistencia. Se dan enfrentamientos en el escenario social que ponen en cuestionamiento los mandatos disciplinarios; esto implica nuevas subjetividades y nuevas modalidades de hacer. Hacer que implica un grado mayor de autonomía, dado el repliegue del Estado de sus antiguas funciones.

Desde este lugar, podemos decir que toda crisis social es también una crisis de identidad; en palabras de Foucault, "todas estas luchas actuales se mueven en torno a la cuestión ¿quiénes somos? Son un rechazo a la violencia estatal, económica, ideológica, que ignora quiénes somos individualmente y también un rechazo de una inquisición científica que determina quién es uno" (Foucault 1988:155).

Surge así, una práctica social que se viene desarrollando en el país, cuyo conjunto se denomina "empresas recuperadas" o "cooperativas autogestionadas", en el marco de lo que se consideran "nuevos movimientos sociales". Son grupos sociales, que afectados por la crisis económica y social comienzan a desarrollar una serie de prácticas autogestivas y autónomas que apuntan a generar relaciones productivas innovadoras y, sobre todo, a lograr un espacio de reconocimiento e identidad en la sociedad.

La autogestión es una posición social de los sujetos frente al proceso productivo y los medios de producción, modelo o proyecto en el cual la empresa y economía están dirigidas directamente por sus trabajadores. En la autogestión no hay dueño, no hay patrón que permita o deje de permitir la participación, sino que la totalidad de los miembros de una empresa asumen su dirección y administración. 
De esta manera, no cambian los objetivos de eficiencia o eficacia, lo que cambia es el régimen por el cual se pretende alcanzarlos, es decir, la participación de todos en la toma de decisiones para la empresa.

Estas prácticas que implican la recuperación de las empresas se instalan con fuerza en el país entre los años 2001 y 2003 y son protagonizadas por trabajadores que, ante la falencia empresarial, sólo les queda la opción del desempleo o la ocupación de la empresa. Así, gran cantidad de empresas son recuperadas por sus trabajadores con el objetivo de defender sus fuentes de trabajo y mantenerlas en funcionamiento. Estas empresas representan uno de los emergentes más originales de la lucha de los trabajadores por conservar sus puestos de trabajo frente a la sistemática destrucción del aparato productivo y como una propuesta frente a la crisis.

De esta manera, desde los aspectos identitarios, nos encontramos con sujetos en lucha, que rápidamente tomaron protagonismo, capacidad de decisión y de acción, logrando así establecer nuevas estrategias de contacto con el mundo. No redujeron su escenario sino que lo ampliaron; ya que si bien conservaron el grupo laboral de pertenencia, lo que les permitió sostener algo de su identidad previa a la recuperación, al mismo tiempo redefinieron espacios laborales y simbólicos, articulándolos con nuevas prácticas y proyectos, estableciendo nuevos lazos de negociación con diferentes actores sociales y políticos.

Ampliaron, además, su dimensión temporal al enfocarse tanto en los aspectos cotidianos, como en proyectos a futuro. Esto fue posible a partir de lograr configurar posicionamientos subjetivos con voluntad de autonomía en sus decisiones.

Sus actuales protagonismos ponen en juego un capital simbólico y cultural que ni ellos mismos suponían tener. De otro modo, no podrían haber emprendido las negociaciones y definición de acciones como las que llevan a cabo en la actualidad.

En definitiva, hemos observado que los cambios operados en las prácticas de los trabajadores de estas nuevas formas de resistencia conllevan un cambio subjetivo. Esta transformación en la subjetividad no implica meramente una modificación de tareas a realizar sino, más bien, un cambio de posición con respecto a sí mismos, a sus pares, 
a la organización y a las funciones que deben desempeñar allí y que les exige un manejo de códigos y de prácticas que por su experiencia anterior les resultaban ajenas (ejercicio de autoridad, organización de trabajo, posicionamiento ético-institucional, toma de decisiones individuales y colectivas, negociaciones políticas, comerciales, legales y sindicales). El cambio en cuanto al posicionamiento subjetivo se evidencia también frente al otro social.

Esta subjetividad en transformación implica haberse constituido en nuevos actores sociales y donde lo que cobra relieve es la ausencia, en la actualidad, de una patronal que decida por ellos. Así, en estos trabajadores, se revela cierta satisfacción por la libertad percibida a pesar del esfuerzo que conlleva el trabajo diario. Esto impacta a su vez, en mecanismos identificatorios con sus pares, en contraposición al esquema jerárquico verticalista de antaño que los marcaba. Si ahora "todos son iguales", el respeto, la igualdad, la participación y las decisiones grupales, les otorgan otra posición subjetiva, proponen nuevas relaciones de poder y nuevos espacios identitarios. En ese sentido, dichos espacios involucran de igual manera, obstáculos, dificultades como así también satisfacciones y alegrías.

Por otro lado, pudimos observar, también, que el significante "Empresa Recuperada" los aglutina y los identifica (trabajadores socios, no socios, profesionales, etcétera), incluso como movimiento social del país. Una identidad construida colectivamente desde muchos sentimientos como vergüenza, impotencia, enojo y hasta deseos de venganza. Pero también, desde el "hacer" y no desde la pasividad, que los define como colectivo social. Una identidad invadida por muchos temores, incertidumbre, pero también una identidad que hizo de la autogestión su fortaleza y que se construye en un discurso donde aparecen las ideas de compromiso y esfuerzo solidario.

Paralelamente, hemos advertido que existen ciertos impedimentos en la dimensión identitaria aludida, que atribuimos a las dificultades que conlleva cualquier proceso que implique una subversión en el poder que otorga un mandato. Estos inconvenientes tienen que ver, primordialmente con la imposibilidad de nombrarse "dueños" o "propietarios" de la empresa. No cuentan con un edificio que les permita acceder al universo de reconocimiento sobre el espacio propio. 
En relación a la categoría poder, observamos un gran temor, por parte de los trabajadores, a retroceder en sus logros y volver a la situación previa a la recuperación de la Clínica. Esto ha generado la negativa a incorporar nuevos socios o a que los profesionales participen y "les invadan" las decisiones. De lo anterior se observa una doble consecuencia.

Por un lado, la imposibilidad de seguir avanzando hacia un espacio cada vez más abierto de planificación y democratización. En ese sentido, los sujetos perciben a las asambleas a partir de dos riesgos: una sólo reunirse y no poder salir del: ¿y ahora qué?, y la otra posibilidad tiene que ver con que se rigidice el mecanismo de la asamblea, es decir, que no se produzca el flujo de relaciones, produciéndose cierta hegemonía de poder que impida el movimiento de los componentes de la relación. Cuestión que, como se observa, tiene implicancias éticas.

Por el otro lado, la necesidad de lograr un espacio discursivo sin contaminaciones de otros discursos del sistema social los tiene, en cierto modo, "atrincherados" y endogámicos. Este discurso endogámico es el que les otorga, al mismo tiempo, la idea de un "saber" que utilizan para evaluar los objetivos no alcanzados. Sin embargo, dichas evaluaciones, muchas veces, terminan por ser parciales y sesgadas.

Las anteriores consideraciones nos llevan a reflexionar sobre ciertos aspectos ético-institucionales involucrados en esta experiencia. Sobre todo si tenemos en cuenta la posición foucaultiana acerca de la ligazón entre las dimensiones del poder y del saber y el atravesamiento de lo ético (Foucault, 1989).

Desde esta perspectiva, el dominio de las prácticas, es decir, lo que tiene que ver con el saber (las prácticas discursivas), del poder (las relaciones con los otros) y de la ética (las relaciones del sujeto consigo mismo), en lo que tienen de específico, se entrelazan.

Así, observamos que el temor y la desconfianza han generado en estos trabajadores ciertos discursos hegemónicos con la finalidad de concentrar poder, pero que descuida la posibilidad de abrir el espacio institucional y por ende, hacerlo más democrático.

De este modo, la dimensión de lo ético institucional cobra una dimensión preponderante, puesto que si tenemos en cuenta 
que concierne a la ética el hecho de involucrar a sujetos que necesariamente ponen en funcionamiento orientaciones valorativas, a la hora de tomar decisiones, la reflexión y concepción de la moral institucional incide de modo decisivo en las prácticas del trabajo institucional. Cuestión esta que, a su vez, refleja un estilo de compromiso y un modo de implicación con la tarea.

A partir de aquí, es que consideramos de importancia la posibilidad de aportar elementos que permitan desarrollar, implementar y gestionar acciones que impacten institucionalmente y los ayuden en la consolidación y afianzamiento de lo emprendido.

\section{Estrategias de intervención implementadas}

Durante el año 2008, en continuidad con el trabajo de investigación anteriormente descrito, se llevó a cabo un proyecto "Beca de extensión de la Universidad Nacional de Córdoba".

Partiendo del objetivo institucional, de tipo comunitario, que se habían propuesto los miembros de la "Cooperativa de Trabajo de la Salud Junín Ltda." a lo largo de estos cinco años, desde la recuperación de la clínica, el cual planteaba: "Retribuir la solidaridad brindada por la sociedad, a través de campañas comunitarias en sectores vulnerables, comedores, merenderos, etcétera"; y ante las diversas dificultades e impedimentos que encontraron para su logro, es que nos planteamos generar un programa que ayude directamente a que los trabajadores cuenten con una planificación y organización de actividades, de manera sistemática, para que por medio del mismo los grupos sociales vulnerables reciban distintos tipos de asistencia.

Una de las primeras dificultades observadas vinculadas a las actividades de acción solidaria que se habían concretado, hasta el momento, se correspondían con la desorganización y la discontinuidad en la asistencia, motivo por el cual no estaban pudiendo cumplir con el objetivo planteado.

La razón de esta situación respondía al crecimiento progresivo de la Cooperativa, ante el cual los trabajadores se vieron sobrepasados por cuestiones operativas y administrativas que demandaban tiempo, esfuerzo, personal a cargo y principalmente, recursos económicos que, desde ya, son escasos. Este crecimiento obligó 
a los trabajadores a cubrir los puestos de trabajo que se iban generando y a postergar las campañas solidarias.

Las campañas comunitarias, destinadas a diversos grupos vulnerables de la ciudad de Córdoba, al comienzo tenían cierta regularidad; los trabajadores brindaban apoyo a diferentes comedores comunitarios ofreciendo y acercando alimentos, juguetes, organizando actividades recreativas para niños, etcétera.

También, realizaban un control sanitario de los diferentes grupos, estableciendo un consultorio por un día en el lugar (villas, comedores, escuelas carenciadas, etcétera) y luego, si era necesario, ponían a disposición los servicios de la clínica para el seguimiento y tratamiento de alguna patología específica.

La jerarquía otorgada, por parte de los trabajadores, a estos proyectos comunitarios, radica en que conciben al fortalecimiento de lazos sociales como el modo que tienen de anudar los vínculos y de retribuir la solidaridad que recibieron de la sociedad. Asimismo, consideran que la aproximación e intercambio, con estos grupos de sectores vulnerables, viabiliza la construcción de redes sociales, que se oponen al encierro y aislamiento propio o característico de otras modalidades de organizaciones en salud (Pymes, empresas privadas, etcétera). Sin embargo, como destacamos, este tipo de acciones no estaba dando el resultado esperado.

Fortalecer el apoyo, a quienes han sido los que se propusieron y los que promovieron esta idea, tuvo como fin lograr, mediante el trabajo en forma conjunta, la organización, efectivización de sus objetivos, ampliando su alcance, dotándolo de mayor solidez y consistencia, afianzándolo y extendiéndolo a otros sectores vulnerables.

En principio, es interesante destacar que durante la primera etapa, en la que encauzamos nuestros esfuerzos en pos de insertarnos en la clínica del mejor modo, logramos tal objetivo a partir de la puesta en marcha de la creación de un dispositivo institucional desde el cual operar.

Respetando los tiempos institucionales, y dando cuenta del dispositivo institucional mencionado, es que comenzamos nuestro trabajo. Desde allí fue posible reconocer los intereses propios de la institución, las preferencias en cuanto al hacer en relación a las campañas comunitarias, para luego en un trabajo conjunto con los 
trabajadores, llevar a cabo una evaluación y selección de aquellas que consideramos más ajustadas a tales intereses en juego y a los recursos disponibles.

Consideramos que las acciones realizadas nos permitieron, además, lograr un trabajo en red coordinado, pensado desde lo común y fundamentalmente, lo posible. De este modo, trabajamos conformando un equipo de trabajo constituido por distintos profesionales pertenecientes a diversas especialidades del campo de la salud, comprometidos y dispuestos a participar activamente, al que ayudamos a organizar lo suficiente, dotándolo de solidez y permanencia.

Entendemos que las actividades realizadas dentro de la clínica, desde el dispositivo creado y a través de las técnicas empleadas, apuntaron al despliegue de aquello que ya estaba allí presente. De allí que intentamos no confundir nuestros posibles aportes con imposiciones ni intervenciones "quirúrgicas".

La operativización, por medio de programas y planes de acción adecuados, logró que la labor que los trabajadores realizaron de manera desorganizada, pudiera ser llevada a cabo y procurase un impacto institucional mayor.

\section{Reflexiones finales}

Como pudimos distinguir, estas nuevas subjetividades o nuevos movimientos sociales suponen nuevos modos de hacer y de funcionar. Una de sus implicancias es intentar atender ciertas necesidades de la sociedad, dando cuenta del establecimiento de una postura ética reflexionada y asumida.

Entendemos que realizar un cálculo a partir de un estudio y análisis minucioso es una instancia pertinente, pero fundamentalmente necesaria para intervenir estratégicamente. Puesto que a partir de los resultados obtenidos, mediante la investigación previa, pudimos intervenir posteriormente, de tal manera que logramos generar un crecimiento de envergadura de la clínica en su interior. Esto a su vez posibilitó la apertura a la comunidad y el fortalecimiento de los lazos, dando lugar a una mayor productividad y bienestar en los trabajadores. 
Dando cuenta de un dispositivo institucional, fue posible realizar intervenciones ajustadas y precisas a quienes iban dirigidas; con claros y concertados modos de inserción en la cooperativa, el dispositivo creado constituyó un lugar simbólico eficaz que dio apertura al desarrollo concreto del proyecto-beca.

El desarrollo de las estrategias pensadas con anterioridad, debieron necesariamente ser replanteadas y modificadas, con el firme propósito de atender las demandas manifiestas, como así también y más especialmente las tácitas.

Respetar la perspectiva de la Institución significó asumir un rol acorde a las necesidades mencionadas, tanto aquellas generales como las específicas de los miembros integrantes. No imponiendo nuestros propios modos de pensar la práctica y en ocasiones alejándonos considerablemente de lo planteado metodológicamente, es que dimos el espacio necesario para el surgimiento de las particularidades propias de la institución y de los profesionales que se desempeñan en ella.

El posicionamiento ético que ante el encuentro y la contingencia permitió el despliegue novedoso y creativo de reestructuraciones que dieron por resultado productos de inestimable valor.

Sabiendo que la reflexión ética gira en torno al fenómeno de la moralidad que regula y legaliza las relaciones humanas, y a partir de lo estudiado y transmitido aquí, es que pensamos que la identidad de los trabajadores de la Cooperativa, se sostiene a partir de un posicionamiento eminentemente ético que es importante subrayar.

Para finalizar, tomamos una frase del texto "Prevención y Psicoanálisis. Propuestas en salud comunitaria" de Moise, que nos permite sintetizar lo logrado por este trabajo: "La posibilidad que la comunidad intervenga en procesos de reparación permite la integridad de un sujeto. Un individuo que circula en los niveles simbólicos de las relaciones sociales es un actor social con mayores recursos psicológicos para una acción específica capaz de transformar sus condiciones de existencia" (Moise, 1998). 


\section{Referencias bibliográficas}

Foucault. M. (1988). El Sujeto y el poder en Dreyfus y Rabinow. Más allá del estructuralismo y la hermenéutica. México: UNAM.

Foucault, M. (1989). Vigilar y Castigar. Buenos Aires: Siglo XXI.

Lewkowicz, I. (2002). Sucesos argentinos. Cacerolazos y Subjetividad postestatal. Buenos Aires: Paidós.

Moise, C. (1998). Prevención y Psicoanálisis. Propuestas en salud comunitaria. Buenos Aires: Paidós. 